\title{
Passer de la connaissance à l'action pour résoudre des problèmes complexes : aperçu de l'examen de neuf cas internationaux
}

B. L. Riley, Ph. D. (1); K. L. Robinson, Ph. D. (2); J. Gamble, MM (3); D. T. Finegood, Ph. D. (4); D. Sheppard, M. Sc. S. (2); T. L. Penney, M.A. (5); A. Best, Ph. D. (6)

Cet article a fait l'objet d'une évaluation par les pairs.

Diffuser cet article sur Twitter

\section{Résumé}

Introduction: La résolution de problèmes complexes du type de la prévention des maladies chroniques présente des défis particuliers pour la création et le transfert de connaissances, soit le passage de la connaissance à l'action (PCA). Les approches axées sur le PCA respectant les principes de la pensée systémique sont jugées prometteuses, mais les stratégies pour les mettre en place ne sont pas bien comprises. Dans cet article, nous présentons les résultats d'une analyse de plusieurs approches systémiques axées sur le PCA dans le but de déterminer comment optimiser leur mise en œuvre et leur efficacité.

Méthodologie: Un groupe consultatif de cinq personnes a choisi neuf initiatives représentant une diversité d'enjeux et de formes organisationnelles. Pour chaque cas, l'information a été recueillie à partir de documents et par l'entremise d'entrevues téléphoniques avec des personnes-ressources de chaque organisation. Après la vérification des descriptions de cas, une analyse inductive a été effectuée à la fois pour chaque cas et entre les cas.

Résultats: Ces cas ont révélé cinq lignes directrices permettant de passer de la conception de systèmes de PCA à leur mise en œuvre : 1) établir et entretenir des relations, 2) coproduire et organiser les connaissances, 3) créer des boucles de rétroaction, 4) les encadrer comme des interventions systémiques plutôt que comme des projets et 5) envisager des variations dans le temps et selon les lieux.

Conclusion: Les résultats de cette analyse contextuelle constituent un départ modeste pour transformer en résultats concrets les concepts systémiques de PCA. L’utilisation des stratégies mises au jour pourrait améliorer le PCA pour la résolution de problèmes complexes en matière de santé publique. Les stratégies elles-mêmes pourront bénéficier de l'évolution d'une science visant à comprendre l'adaptation et l'apprentissage constant des politiques et des interventions pratiques, ce qui renforcera les relations durables et comblera les lacunes des systèmes, et pas simplement celles des données probantes. Les approches systémiques de PCA tireront également des bénéfices d'évaluations rigoureuses.

Mots-clés : études d'intervention, santé publique, transfert de connaissances, approche systémique
Principales constatations

- La résolution de problèmes complexes comme la prévention des maladies chroniques nécessite de partager et d'utiliser les connaissances sur ce qui fonctionne et comment dans le soutien aux changements positifs dans les collectivités.

- Notre analyse de neuf exemples variés utilisant le passage de la connaissance à l'action a établi qu'il n'existe pas de formule ou de recette unique pour appliquer des connaissances à des problèmes précis; que les solutions doivent surgir et s'adapter au fil du temps en fonction des rétroactions et des évaluations; que des relations à long terme devraient être nouées et entretenues, mettant l'accent sur le partage des ressources pour faciliter la pensée créatrice et les solutions et enfin que nous devons ensemble bâtir et utiliser de nombreux types de connaissance dans tous les secteurs.

\section{Introduction}

Les problèmes de santé publique, dont fait partie la prévention des maladies chroniques, surviennent et demeurent en raison de multiples facteurs physiologiques, comportementaux et environnementaux et de leurs interactions ${ }^{1,2}$. Les solutions à ces problèmes complexes nécessitent de

Rattachement des auteurs :

1. Propel Centre for Population Health Impact, Université de Waterloo, Waterloo (Ontario), Canada

2. Agence de la santé publique du Canada, Ottawa (Ontario), Canada

3. Imprint Consulting, Hampton (Nouveau-Brunswick), Canada

4. Université Simon Fraser, Burnaby (Colombie-Britannique), Canada

5. Cambridge University, Cambridge, Royaume-Uni

6. InSource Research Group, Vancouver (Colombie-Britannique), Canada

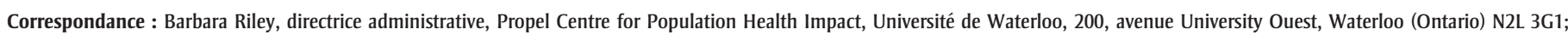
tél. : 519-888-4567, poste 37562; téléc. : 519-746-8171; courriel : briley@uwaterloo.ca 
nouvelles approches sur la façon dont les connaissances sont établies et appliquées ${ }^{3-5}$. Dans cet article, nous les appelons « approches systémiques du passage de la connaissance à l'action [PCA]».

Les approches systémiques du PCA se fondent sur des approches linéaires qui mettent l'accent sur une présentation et une diffusion efficaces des produits de la connaissance et sur des approches en matière de relations qui reconnaissent l'importance de partenariats efficaces pour acquérir et partager les connaissances. Les approches systémiques conviennent du fait que les processus de diffusion comme les relations elles-mêmes sont façonnés, intégrés et organisés dans des structures qui influencent les types d'interactions survenant entre de multiples intervenants ayant des visions du monde, des priorités, des langages, des moyens de communication et des attentes différents ${ }^{6}$. Ces intervenants appartiennent tous à un système (lui-même façonné par la culture, les structures, les priorités et les capacités ${ }^{7}$ ) qui demande à être activé pour que ses différentes parties soient connectées. Penser en termes de systèmes est donc nécessaire pour activer le $\mathrm{PCA}^{8,9}$.

Même si on sait qu'il faut disposer d'approches systémiques du PCA et même si ce concept gagne en clarté, les stratégies pratiques de conception et de mise en œuvre d'approches systémiques du PCA sont rares $^{8,10,11}$. L'objectif de l'analyse contextuelle présentée dans cet article était de commencer à offrir des stratégies pratiques utiles pour concevoir, mettre en œuvre et rendre efficaces des initiatives de PCA afin de résoudre des problèmes complexes.

Les maladies chroniques représentent un problème grave et urgent partout dans le monde. En 2005, 60 \% des décès dans le monde étaient attribuables aux maladies chroniques et une hausse de $17 \%$ était prévue entre 2005 et $2015^{12}$. Étant donné qu'on estime que jusqu'à $50 \%$ des cancers $^{13}, 90 \%$ des maladies cardiovasculaires $^{14}$ et $91 \%$ des cas de diabète ${ }^{15}$ sont évitables, les investissements importants et croissants dans la prévention à l'échelle de la population se révèlent encore insuffisants. Pourtant, même des réductions modestes dans les facteurs de risque de maladie chronique contribue- raient à sauver des dizaines de milliers d'années de vie et à économiser des centaines de millions de dollars en coûts directement liés aux soins de santé dans le monde chaque année $\mathrm{e}^{16,17}$.

Par ailleurs, les maladies chroniques constituent des problèmes complexes. Cette complexité est attribuable à une interaction dynamique entre facteurs contribuant au développement et à la persistance des états chroniques. Ces facteurs sont variés, qu'ils relèvent de la physiologie et du comportement individuels, des arrangements institutionnels (p. ex. les interactions entre organismes de santé et de services sociaux) ou des environnements physiques et sociaux à l'échelle locale comme sociétale (p. ex. aménagement des quartiers, cohésion sociale, approvisionnement et distribution alimentaires $)^{18}$.

Les problèmes complexes que constituent les maladies chroniques ne peuvent donc pas être réglés par des solutions simples, isolées et ponctuelles. Ils nécessitent, pour être résolus, des interventions coordonnées et des actions créatives à de nombreux niveaux administratifs (p. ex. régional, provincial, national et international) ${ }^{4}$ et systémiques (p. ex. paradigmes, objectifs, structures organisationnelles) $)^{5,19}$. Ils nécessitent aussi la mobilisation des intervenants et des organisme travaillant dans les domaines de la recherche, des politiques et des pratiques, à la fois dans les secteurs de la santé et dans d'autres secteurs. Ils nécessitent de plus que les interventions en matière de politiques et de programmes soient adaptées aux divers contextes et que ces adaptations à des environnements dynamiques se fassent en permanence ${ }^{4}$, toujours dans le but de modifier les comportements liés à la santé et les facteurs sous-jacents de risque liés aux maladies chroniques.

Trouver des solutions polyvalentes et dynamiques aux problèmes complexes constitue un défi particulier à l'acquisition et au transfert des connaissances ${ }^{20-23}$. Par exemple, il est maintenant largement reconnu que l'application du concept d'une médecine fondée sur les données probantes tel qu'il a été conçu à l'origine n'est pas adaptée aux interventions en santé publique $^{24}$, ce qui rend problématique l'application directe des résultats de recherche à des contextes, des périodes et des populations variés. Des approches de PCA efficaces devront au contraire constituer des processus dynamiques et multidirectionnels mobilisant les intervenants (souvent variés) à créer conjointement, à synthétiser, à partager et à utiliser des connaissances afin d'éclairer la prise de décision et de favoriser ainsi le changement en fonction des contextes et des besoins liés aux politiques et aux programmes ${ }^{25}$.

Dans cette optique, de nouvelles approches en santé publique fondée sur des données probantes ont été explorées et ont mené à de nombreux appels en faveur de l'application des concepts et des méthodes de la science des systèmes ${ }^{8,23,26-30}$. Les réponses à ces appels ont donné lieu à un nombre croissant d'études empiriques utilisant des techniques de modélisation systémique et d'analyse de réseaux. Ces études empiriques ont également contribué à l'émergence d'une littérature sur les potentialités de l'application de la pensée systémique, définie de façon variable, au développement et à l'utilisation des connaissances au sein de la population et en santé publique ${ }^{10,26-28,31,32}$. Cette documentation demeure cependant relativement silencieuse sur les modalités de transformation des concepts et des principes de la pensée systémique en stratégies pratiques conduisant à la création et à l'utilisation de connaissances destinées à la résolution de problèmes complexes.

Des organismes comme l'Agence de la santé publique du Canada ou le Propel Centre for Population Health Impact, qui ont un mandat pancanadien pour accélérer le PCA dans la prévention des maladies chroniques, tentent de combler cette lacune. L'analyse et la synthèse présentées dans cet article sont ainsi fondées sur une série d'initiatives s'attaquant à des problèmes complexes, axées sur la création et l'utilisation de connaissances et appliquant les principes de la pensée systémique dans leurs tentatives de PCA.

\section{Méthodologie}

Les membres d'un petit groupe consultatif (les auteurs BR, KR, DF, DS, AB), représentant divers organismes de recherche ou responsables de politiques en matière de santé publique ou de PCA et ayant déjà 
TABLEAU 1

Principes de la pensée systémique pour résoudre les problèmes complexes de santé publique

\begin{tabular}{|c|c|c|}
\hline Cohérence & Connectivité & Apprentissage constant \\
\hline Harmoniser capacités et complexité & Former des réseaux et des équipes & Éviter un paradigme réducteur \\
\hline $\begin{array}{l}\text { Agir localement, communiquer régionalement, } \\
\text { apprendre globalement }\end{array}$ & Soutenir les individus & Fixer des objectifs fonctionnels \\
\hline Leadership favorisant le changement & Établir une confiance authentique & Évaluer l'efficacité \\
\hline Encourager les innovations perturbatrices & Répartir le pouvoir de décision et d'action et l'autorité & Favoriser un processus fondé sur les relations et les échanges \\
\hline
\end{tabular}

collaboré à des projets liés au PCA pour la prévention des maladies chroniques, ont choisi trois critères principaux pour sélectionner les organismes et leurs initiatives en matière de PCA : (1) intermédiaires (par opposition à des organismes de prestation de services directs) abordant un enjeu complexe, (2) groupes cherchant à intervenir à de multiples niveaux dans un système et (3) groupes ayant utilisé des stratégies précises en matière de PCA en utilisant la pensée systémique.

Les principes de la pensée systémique englobent une vaste gamme de disciplines et d'écoles de pensée. Pour notre analyse, nous avons intégré des idées provenant de plusieurs penseurs systémiques ${ }^{3,34-36}$ et nous les avons adaptées au contexte de la santé publique. Grâce à des discussions et un consensus au sein des membres de notre groupe consultatif, nous avons adopté 12 principes systémiques valables pour résoudre les problèmes complexes en matière de santé publique et nous les avons classés selon 3 concepts généraux : la cohérence, la connectivité et l'apprentissage constant (voir le tableau 1).

En utilisant les 3 critères de sélection de cas, nous avons établi une liste de 30 cas se rapportant au PCA, grâce à une recherche sur Internet et une recherche parmi les articles, publiés ou appartenant à la littérature grise, qui explorent les approches complexes ou systémiques du PCA, et également en demandant à certaines personnes connues des membres du groupe consultatif de soumettre quelques propositions. Tous les cas de la liste initiale concernaient le Canada ou d'autres pays développés.

À partir de l'analyse des documents disponibles pour le public, nous avons choisi les 9 cas (parmi les 30 cas initiaux) qui correspondaient le mieux à nos critères et à la portée de notre analyse (voir le tableau 2). Nous avons ainsi exclu les cas d'organismes offrant une prestation directe de services, n'intervenant qu'à un seul niveau d'un système (p. ex. ne s'intéressant qu'au changement de comportement individuel) ou n'ayant pas adopté

TABLEAU 2

Organismes ciblés par l'analyse

\section{Organisme}

Tamarack Institute - Vibrant Communities Canada (Toronto, Ontario, Canada) http://tamarackcommunity.ca

Framework (Toronto, Ontario, Canada) http://www.frameworkorg.org

Plexus Institute (Washington, DC, É.-U.)

http://www.plexusinstitute.org

Centre of Excellence in Intervention and Prevention Science (CEIPS) (Victoria, Australie) http://ceips.org.au/

INSPIRE's EPISCentre (université de l'État de Pennsylvanie, É.-U.)

http://www.episcenter.psu.edu/

ResearchImpact-RéseaulmpactRecherche (RIR) (Canada)

http://www.researchimpact.ca/localRI/YorkU/

Social Innovation Generation (SiG) Causeway (Ontario)

http://www.sigeneration.ca

Centraide Toronto (Ontario, Canada)

http://www.unitedwaytoronto.com/

Stratégie nationale sur le traitement - réseau SystemAction (Canada) http://www.nts-snt.ca/
Type d'initiative de passage de la connaissance à l'action

Intermédiaire qui place l'apprentissage ainsi que la production et la diffusion de connaissances au centre d'une initiative de portée nationale visant la réduction de la pauvreté

Petite organisation créative qui a réalisé un projet pilote avec plusieurs autres organismes afin d'explorer comment des outils technologiques aisément accessibles peuvent aider ces organismes à recueillir et à mettre en commun leurs données, leurs pratiques et leurs connaissances

Organisation de renforcement des capacités et d'action-recherche explicitement orientée sur la notion de complexité et sur la pensée systémique

Exemple de centre de recherche en santé publique

Exemple où un outil Internet de collecte et de diffusion des données constitue la pièce maîtresse des activités de PCA

Exemple de l'unité de PCA d'une université canadienne faisant aussi partie d'un projet de collaboration interuniversitaire visant à promouvoir l'application de la recherche aux politiques et aux pratiques

Exemple de divers organismes collaborant sur un thème commun

Orgáanisation intermédiaire qui a misé sur une approche utilisant la communauté de pratiques pour mobiliser les connaissances en matière d'éducation des jeunes

Réseau et stratégie de portée nationale visant l'application d'une approche systémique dans les interventions relatives aux lacunes des services de traitement de l'alcoolisme et des toxicomanies et aux besoins des clients à tous les échelons gouvernementaux et dans divers types d'organismes 
d'approche du PCA (p. ex. certains organismes ont produit des documents de discussion sur des concepts ou des théories d'approches systémiques mais n'ont mis en branle aucune initiative précise).

Les 9 cas sélectionnés sont variés, portent sur des secteurs différents et correspondent à diverses formes organisationnelles. Dans certains cas, le PCA représentait un objectif fondamental (p. ex. pour RéseauImpactRecherche et pour Plexus) alors que dans d'autres cas il s'agissait d'une activité auxiliaire à l'appui d'autres objectifs (p. ex. pour la Stratégie nationale sur le traitement et pour Causeway). Certains employaient explicitement un cadre systémique ou axé sur la complexité (Tamarack, Plexus et le CEIPS), tandis que d'autres comportaient des particularités compatibles avec une approche systémique sans utiliser directement ce langage ou cette structure (p. ex. INSPIRE et RéseauImpactRecherche). Ainsi, que ce soit explicitement ou implicitement, toutes les activités de PCA de ces organismes étaient fondées sur les principes de la pensée systémique.

Une série de questions a été mise au point pour guider la documentation et l'analyse. Elle a porté sur la vision organisationnelle, sur les objectifs, sur les principes directeurs, sur les collaborateurs, sur les activités, sur les définitions opérationnelles, sur les résultats et sur les leçons retenues. La cueillette des données de départ a reposé sur l'analyse de la documentation rendue publique (rapports ou publications et renseignements provenant de sites Internet) et sur une entrevue téléphonique, pour chacun des organismes, avec une personne-ressource bien informée au sujet de chaque cas (p. ex. directeur administratif ou bien chef de projet ou coordinateur). Ces personnes-ressources ont vérifié les descriptions de cas et ont fourni des renseignements supplémentaires, soit directement soit en recommandant des rapports que les membres du groupe consultatif n'avaient pas encore consultés.

Nous avons effectué un examen inductif de la documentation de chaque cas en nous référant aux principes systémiques présentés dans le tableau 1, sans toutefois nous laisser limiter par eux. Ces principes ont servi de cadre analytique préliminaire pour examiner comment ils ont été mis en œuvre dans un ou plusieurs cas. Des thèmes relatifs aux stratégies pratiques (p. ex. personnes, processus, structures) ont été générés et codés dans la documentation de chaque cas. L'analyse a été effectuée par deux chercheurs (les auteurs JG et TP) et a été complétée par les réflexions et les analyses du groupe consultatif à partir de l'examen des exemples documentés pour chaque thème. Nous avons ensuite comparé les thèmes pour faire état de leurs similarités et de leurs différences puis nous avons sélectionné quelques exemples précis de thèmes touchant plusieurs cas.

\section{Résultats}

À partir de notre analyse, nous avons pu établir cinq lignes directrices sur la mise en œuvre d'approches systémiques du PCA :

\section{1) Établir et entretenir des relations}

La création de relations, exprimée sous différentes formes organisationnelles (p. ex. réseaux, coalitions, groupes consultatifs), constituait un thème central commun à tous les cas. La qualité et la profondeur de ces relations, au-delà du partage de l'information, étaient visibles. Le fait d'avoir des conseillers, des partenaires et des employés ayant acquis de l'expérience au sein des secteurs gouvernemental, privé et bénévole aide à avoir accès aux différentes personnes et aux différents groupes, à acquérir des compétences en gestion des relations et à comprendre les nuances du langage et de l'interprétation. Par exemple, l'un des principes de base de l'initiative Vibrant Communities du Tamarack Institute consistait à établir des collaborations multisectorielles au sein des collectivités et à fournir des mesures de soutien pour alimenter et soutenir ces collaborations. L'éventail de perspectives offertes par les organismes bénévoles, les dirigeants d'entreprise, les représentants gouvernementaux et les personnes ayant vécu dans la pauvreté a engendré plusieurs solutions potentielles et suscité l'adhésion aux stratégies élaborées ensuite.

\section{2) Coproduire et organiser des connaissances}

Dans tous les cas étudiés, il était clair que le PCA pour les problèmes complexes exigeait une interprétation, une analyse et une logique communes. Les paradigmes d'expert quant à la création et à la diffusion des connaissances ne sont d'aucune utilité dans le domaine du complexe. Si nous faisons peu de cas des personnes qui suggèrent des améliorations aux pratiques ou si ces personnes n'ont pas le temps ou l'occasion de valider leurs idées, le potentiel de changement est limité. La métaphore de l'organisation est utile dans quelques cas lorsque nous pensons aux approches systémiques du PCA. L'organisation consiste à relier des éléments semblables et différents. Il ne s'agit pas seulement de demander aux gens ce qu'ils veulent savoir, il faut anticiper ce qui peut être intéressant et utile pour les politiques et les pratiques mais qui n'a pas forcément été repéré par les groupes concernés. Le CEIPS a par exemple adopté cette approche en intégrant du personnel de recherche dans les comités de son administration sanitaire locale afin d'établir des relations avec les employés chargés des pratiques et des politiques et d'intégrer leurs points de vue plus directement au travail de recherche.

\section{3) Créer des boucles de rétroaction}

Notre analyse des cas a montré que les tentatives de PCA pour les problèmes complexes nécessitent des résultats contextuels qui décrivent ce qui fonctionne (ou ne fonctionne pas) pour qui, comment et dans quel contexte. De nombreux cas intègrent une boucle de rétroaction pour favoriser l'utilisation des connaissances pour orienter l'action. Cela requiert une évaluation réflexive : les activités de PCA sont prises en compte et l'on cible la révision des actions. De telles boucles de rétroaction contribuent à produire des données factuelles pertinentes, qui sont mises à la disposition des organismes s'occupant des politiques et des pratiques. Par exemple, INSPIRE a créé un environnement virtuel permettant à divers intervenants d'avoir accès à des données et à des renseignements à partir de différentes sources et de les intégrer afin de soutenir l'amélioration continue de la qualité dans la prestation des interventions reposant sur des bases empiriques. INSPIRE a également facilité la cueillette de données normalisées auprès de nombreuses institutions, créant ainsi une source de données importante pour l'étude de la mise en œuvre et des résultats au sein des divers organismes. 


\section{4) Encadrer selon les modalités des interventions systémiques et non celles des projets}

Certains des organismes étudiés ont été contraints de produire des résultats tangibles à court terme. Lorsque nous avons pour préoccupation centrale les processus systémiques et que nous incitons d'autres organismes à combler certaines lacunes, nous devons orienter nos efforts de collaboration vers la recherche de nouveaux acteurs, susciter l'intérêt pour un plan d'action global et encourager d'autres organismes ayant un mandat à long terme et possédant une expertise pertinente à diriger certaines interventions spécifiques. Par exemple, les intervenants de SiG Causeway ont été attentifs à ne pas créer de structure de gouvernance encombrante ayant pour ambition d'élaborer et de mettre en œuvre une série de projets. Ils ont plutôt créé un réseau central de leaders extrêmement engagés provenant de différents champs, tous guidés par un cadre stratégique global visant à favoriser le leadership intersectoriel et le partage des expériences d'apprentissage afin de faire avancer le financement social au Canada. Ces chefs de file se sont réunis lors d'événements de démonstration et d'apprentissage au Canada, ont organisé des webinaires pour établir des liens entre divers organismes relevant de divers secteurs afin de discuter de financement social et ont contribué à créer et à alimenter un carrefour ou portail Web commun avec des guides, des ressources et des livres blancs pour passer des concepts à l'action.

\section{5) Envisager des types de soutien différents selon les moments et les contextes}

Même si les objectifs sont demeurés les mêmes au sein des organismes étudiés au fil du temps, leurs activités et leurs stratégies en matière de PCA ont souvent changé, s'adaptant aux besoins de leurs partenaires et clients. Ces organismes ont choisi, parmi un vaste éventail d'options de PCA, celles qui étaient le mieux adaptées au moment, à l'endroit, aux personnes et au but poursuivi. Par exemple, RéseauImpactRecherche a construit chaque initiative ayant trait au PCA à partir d'une série d'activités qui avaient été réalisées au fil du temps. De façon semblable, la communauté de pra- tique de Centraide a proposé un éventail d'événements parmi lesquels chacun pouvait choisir ce qui l'intéressait.

\section{Analyse}

Les cinq lignes directrices sur les stratégies pratiques destinées à mettre en œuvre des approches systémiques de PCA confirment l'importance de plusieurs principes systémiques. En premier lieu, l'importance des relations a été confirmée, notamment des relations durables entre personnes et groupes ayant des points de vue différents, que ce soit à propos de la recherche, des politiques ou des pratiques. A également été confirmée l'importance d'adapter les principes aux divers contextes et à leur dynamique, et celle d'utiliser la rétroaction pour un apprentissage constant. Comme nous le souhaitions, nous avons pu relever divers exemples pratiques d'application de ces principes. Nos résultats ne constituent cependant qu'un départ modeste dans le projet de fournir des indications et des orientations utiles afin de mieux traduire en pratique les concepts systémiques du PCA. En s'appuyant sur ces résultats, le groupe consultatif a défini trois orientations prometteuses pour le PCA en vue de résoudre des problèmes complexes.

\section{1) Adopter des approches émergentes et globales}

Les approches systémiques remettent en question les idées reçues au sujet des attentes, des garanties, des réponses finales et du " contrôle », en particulier en ce qui concerne les scientifiques et les spécialistes possédant une expertise particulière. Dans les systèmes complexes et dynamiques, aucun modèle ne demeure en place longtemps, et les résultats des interventions peuvent ne pas être aussi solides que ce que la science considère en général comme souhaitable. Nous devons ainsi éviter les approches fondées sur des formules, tout particulièrement dans des contextes différents. Par exemple, il ne convient pas de répéter à l'identique une intervention réussie dans d'autres collectivités ou populations. Reconnaître que le contexte est important n'empêche cependant pas de relever les composantes essentielles du succès et de tirer des enseignements de divers contextes ou de documenter les conditions favorables au succès, comme ce fut fait par Vibrant Communities de Tamarack et SiG Causeway. Il est nécessaire de mettre au point et d'utiliser des méthodes de recherche et d'évaluation adaptées au contexte pour apprendre ce qui fonctionne, comment et pourquoi. Ces indications permettront ensuite d'adapter les interventions de manière appropriée.

De plus, ces approches émergentes et globales nécessitent un apprentissage constant. Des efforts sont nécessaires pour continuer à procéder à des évaluations et à tirer profit des adaptations ainsi qu'à étudier les processus de mise au point et d'amélioration des interventions ${ }^{37}$. On peut ainsi mettre au point des modèles originaux et participatifs permettant des évaluations ciblées sur l'apprentissage au sein d'environnements dynamiques, et non des évaluations ciblées sur les résultats, construites en référence à la notion de responsabilité et tenant peu ou pas du tout compte du contexte.

\section{2) Mettre l'accent sur des relations durables axées sur les solutions}

Pour être efficace, des relations durables sont nécessaires entre les secteurs des politiques, des pratiques et de la recherche afin de définir adéquatement, mettre à l'essai et adapter des solutions à des problèmes complexes. Dans un contexte de ressources limitées, les activités visant à établir ces relations sont facilement considérées comme un luxe ne méritant ni temps ni financement. Pourtant, le fait d'inclure des phases de développement au financement des projets pourrait constituer un ingrédient essentiel à la mise en place d'interventions efficaces et pertinentes et de méthodes de recherche appropriées pour mobiliser et utiliser les données probantes utiles à la réalisation des changements désirés.

L'analyse des cas de Centraide Toronto et de la Stratégie nationale de traitement montre que l'investissement dans des modèles de communautés de pratique et l'utilisation de ces modèles, même si leurs structures, leurs objectifs et leurs buts sont différents, pourrait également être prometteur dans ce domaine. Ces modèles de 
communautés de pratique - guidés par des principes systémiques - mettraient l'accent sur les questions contextuelles, seraient orientés vers l'apprentissage constant et l'échange diversifié, valoriseraient l'émergence, privilégieraient différentes perspectives et sources de connaissances et enfin viseraient à comprendre le contexte de mise en œuvre. La recherche de collaborateurs et de points de vue diversifiés pour travailler à la résolution de problèmes complexes suppose l'inclusion : le fait de réunir en toute connaissance de cause des personnes qui ne partagent pas nécessairement les mêmes points de vue, une culture commune ou le même langage permet d'aller au-delà des différences. Les rapports de force doivent être reconnus et combattus au sein de ces collaborations (p. ex. comment accorder une voix égale aux participants de races, de sexes, de classes et de scolarité différents). Le temps et les efforts nécessaires pour bâtir des relations durables et diversifiées sont rentables, surtout si l'on veille à ce que ces relations soient établies de façon authentique et solide.

\section{3) Combler les lacunes du système outre celles en matière de données probantes}

Le développement des connaissances en santé publique vise en général à combler diverses lacunes en matière de données probantes, en particulier en fournissant des descriptions détaillées des problèmes, et plus récemment en portant une attention croissante à l'étude des interventions en matière de politiques et de programmes ${ }^{11,38}$. Conformément aux modèles portant sur les lieux où intervenir dans les systèmes complexes $^{3,4}$, les résultats de l'analyse font ressortir comme importants à la fois les changements dans les structures (p. ex. équipes multidisciplinaires, réseaux) et la rétroaction, qui sont dans les deux cas des interventions liées aux systèmes. L'un des grands défis demeure le financement limité accordé au comblement des lacunes liées aux éléments structurels et aux systèmes, par exemple à la rétroaction par l'entremise de méthodes d'évaluation communes entre différents organismes et administrations ${ }^{30}$.

\section{Forces et limites}

Les principales forces de cette analyse relèvent d'une part de l'importance et de la pertinence de ses objectifs pour l'avancement d'un PCA efficace dans le cadre d'enjeux complexes comme la prévention des maladies chroniques et, d'autre part, de la méthodologie utilisée.

Le secteur de la santé publique a accordé beaucoup plus d'importance à la définition des problèmes qu'à l'élaboration de solutions. L'intervention par l'entremise de politiques, de programmes et de changements systémiques pour résoudre les problèmes urgents, graves et complexes en santé publique, en particulier les maladies chroniques, requiert de nouvelles méthodes de travail, en particulier de nouvelles modalités de création et d'application des connaissances qui tiennent compte de la complexité des problèmes. Notre analyse permet de comprendre comment mettre en œuvre dans les pratiques les approches prometteuses de PCA.

La valeur de nos résultats est renforcée par la méthodologie utilisée pour déterminer et sélectionner l'éventail des cas à analyser. Les méthodes d'échantillonnage ont donné un nombre suffisant et gérable de cas pertinents, tout en assurant une diversité convenable. Les résultats sont d'autant plus valables que nous avons utilisé une approche systémique dans la sélection, la cueillette et l'analyse de l'information pour les cas et entre ceux-ci, et que nous avons obtenu cette information grâce à la fois à de la documentation et à des entrevues.

Notre analyse était limitée par sa portée modeste en raison de ressources limitées. Elle était de nature préliminaire et exploratoire par sa conception même. Nos résultats laissent néanmoins penser qu'il y a beaucoup à apprendre au sujet du PAC pour résoudre les problèmes complexes à partir des réalisations existantes. Une étude qualitative et quantitative et une observation plus en profondeur des initiatives de PCA utilisant des approches systémiques permettraient de dégager des conclusions plus approfondies et plus complètes. Il serait également utile de se pencher sur les cas déjà analysés et sur d'autres cas pendant une période plus longue, en particulier pour mieux comprendre les façons de faciliter les processus dynamiques du PCA et leur efficacité dans divers contextes.

\section{Conclusion}

On doit tenir compte, lorsqu'on s'attaque au fardeau des maladies chroniques, de la complexité des problèmes et des environnements connexes, et en particulier de la nature des connaissances nécessaires pour résoudre de tels problèmes ainsi que des modalités et des raisons à l'origine de la création et de l'utilisation de ces connaissances. En s'inspirant d'exemples d'approches systémiques de PCA déjà réalisées, on a pu déterminer diverses stratégies pratiques de mise en œuvre. Ces dernières peuvent être améliorées en mettant l'accent sur une approche globale de la science, en se concentrant sur l'adaptation des interventions, en renforçant les relations durables assurant des points de vue et des apports diversifiés, et en comblant les lacunes du système en plus de combler celles des données probantes. Alors, des progrès au moins modestes pourront être réalisés dans la transformation des environnements, des organismes et des comportements pour résoudre les problèmes complexes en santé publique.

\section{Remerciements}

Ce projet a été financé par l’Agence de la santé publique du Canada. Barbara Riley a reçu une subvention de la Société canadienne du cancer (subvention n²011701019).

\section{Références}

1. Rittel HW, Weber MM. Dilemmas in a general theory of planning. Policy Sci. 1973;4(2) : 155-169.

2. Kreuter MW, De Rosa C, Howze EH, Baldwin GT. Understanding wicked problems : a key to advancing environmental health promotion. Health Educ Behav. 2004;31(4) : 441-454.

3. Finegood DT. The complex systems science of obesity. Dans : Cawley J (dir.), The Oxford handbook of the social science of obesity. Oxford (UK) : Oxford University Press; 2011. p. 208-236.

4. Meadows DH. Thinking in systems : a primer. Wright $\mathrm{D}$, editor. White River Junction (VT) : Chelsea Green Publishing; 2008. 
5. Green LW, Glasgow RE, Atkins D, Stange K. Making evidence from research more relevant, useful, and actionable in policy, program planning, and practice : slips "Twixt cup and lip”. Am J Prev Med. 2009;37(6 Suppl 1) : S187-191.

6. Frenck J. Balancing relevance and excellence : organizational responses to link research with decision-making. Soc Sci Med. 1992;35 : 1397-1404.

7. Best A, Terpstra JL, Moor G, Riley B, Norman CD, Glasgow RE. Building knowledge integration systems for evidenceinformed decisions. J Health Organ Manag. 2009;23(6) : 627-641.

8. Best A, Holmes B. Systems thinking, knowledge and action : towards better models and methods. Evid Policy. 2010;6(2) : 145-159.

9. Best A, Trochim W, Haggerty J, Moor G, Norman CD. Systems thinking for knowledge integration : new models for policyresearch collaboration. Dans McKee L, Ferlie E, Hyde P, (dir.), Organizing and reorganizing : power and change in health care organizations. London (UK) : Routledge; 2008. p. 154-166.

10. Holmes BJ, Finegood DT, Riley BL, Best A. Systems thinking in dissemination and implementation research. Dans : Brownson RC, Colditz GA, Proctor EK (dir.), Dissemination and implementation research in health : translating science to practice. New York (US) : Oxford University Press; 2012. p. 175191.

11. Cameron R, Riley BL, Campbell HS, Manske $\mathrm{S}$, Lamers-Bellio K. The imperative of strategic alignment across organizations : the experience of the Canadian Cancer Society's Centre for Behavioural Research and Program Evaluation. Can J Public Health. 2009;100(1) : Suppl I27-30.

12. Organisation mondiale de la santé (OMS). Rapport sur la situation mondiale des maladies non transmissibles 2010. Genève (CH) : OMS; 2011.

13. Société canadienne du cancer (SCI) et Institut national du cancer du Canada (INC). Statistiques canadiennes sur le cancer 2007. Toronto (Ont.) : SCI et INC.
14. Organisation mondiale de la santé (OMS). Prévention des maladies chroniques : un investissement vital. Genève $(\mathrm{CH})$ : OMS; 2005. Téléchargeable à partir de la page : http://www.who.int/chp/chronic_disease_ report/contents/en/

15. Hu FB, Manson JE, Stampfer MJ et collab. Diet, lifestyle, and the risk of type 2 diabetes mellitus in women. N Engl J Med. 2001; 345(11) : 790-797.

16. Dobrow MJ, Goel V, Upshur RE. Evidencebased health policy : context and utilisation. Soc Sci Med. 2004;58(1) : 207-217.

17. Rose G. The strategy of preventive medicine. Oxford (UK) : Oxford University Press; 1993.

18. Vandenbroek P, Goossens J, Clemens M. Tackling obesities : future choices - building the obesity system map. (UK) : Government Office for Science; 2007. PDF téléchargeable à partir de la page : https://www.gov.uk /government/publications/reducing-obesity -obesity-system-map

19. Lavis JN, Davies HTO, Gruen RL, Walshe $\mathrm{K}$, Farquhar CM. Working within and beyond the Cochrane collaboration to make systematic reviews more useful to healthcare managers and policy makers. Healthc Policy. 2006;1(2) : 21-33.

20. Brownson RC, Fielding JE, Maylah CM. Evidence-based public health : a fundamental concept for public health practice. Ann Rev Public Health. 2009;30 : 175-201.

21. Kiefer L, Frank J, Di Ruggiero E, et al. Fostering evidence-based decision-making in Canada : examining the need for a Canadian population and public health evidence centre and research network. Can J Public Health. 2005;96(3) : 111-119.

22. Riley B, Edwards N. A primer on multiple intervention programs and some implications for a research agenda [Internet]. Ottawa (Ont.) : Centre de recherche sur la santé communautaire; 2009. (n M2009-1). PDF téléchargeable à partir du lien : http:// aix1.uottawa.ca/ nedwards/chru/english /pdf/M2009-1.pdf

23. Hobin EP, Hayward S, Riley B, Di Ruggiero E, Birdsell J. Maximising the use of evidence : exploring the intersection between population health intervention research and knowledge translation from a Canadian perspective. Evid Policy. 2012;8(1) : 97-115.
24. Green LW. From research to "best practices" in other settings and populations. Am J Health Behav. 2001;25(3) : 165-178.

25. Graham ID, Logan J, Harrison MB, et al. Lost in knowledge translation : time for a map. J Contin Educ Health Prof. 2006;26(1) : 13-24.

26. Best A, Clark P, Leischow S, Trochim W, editors. Transforming tobacco control through systems thinking : integrating research and practice to improve outcomes. Smoking and Tobacco Control Monograph 18. Bethesda (MD) : Department of Health and Human Services, Public Health Service, National Institutes of Health, National Cancer Institute, NIH : 2007.

27. Finegood DT, Johnston L, Giabbanelli P et collab. Complexity and systems thinking. In LW. Green (dir.), Oxford Bibliographies Online [Internet]. New York : Public Health; 2012 [consulté le 21 mai 2014]. Consultable à la page : http://www.oxfordbibliographies .com/view/document/obo-9780199756797 /obo-9780199756797-0049.xml?rskey = VJCfVs \&result $=11 \& q=$

28. Riley B, Norman C, Best A. Knowledge integration in public health : a rapid review using systems thinking. Evid Policy. 2012; 8(4):417-32.

29. Kumaniyika SK, Parker L, Sim LJ (dir.). Bridging the evidence gap in obesity prevention : a framework to inform decision making. Washington (DC) : Institute of Medicine, The National Academies Press; 2010.

30. Green LW, Sim L, Breiner $\mathrm{H}$ (dir.). Evaluating obesity prevention efforts : a plan for measuring progress. Washington (DC) : Institute of Medicine, The National Academies Press; 2013.

31. Foster-Fishman PG, Nowell B, Yang H. Putting the system back into systems change : a framework for understanding and changing organizational and community systems. Am J Community Psychol. 2007; 39(3/4):197-216.

32. Leischow SJ, Best A, Trochim WM et collab. Systems thinking to improve the public's health. Am J Prev Med. 2008;35(2S) : S196203. 
33. Gamble J, Penney T. Approches systémiques de la mobilisation des connaissances : analyse des initiatives. Ottawa (Ont.) : Agence de la santé publique du Canada, Division des interventions liées aux maladies chroniques; 2012.

34. Bar-Yam Y. Complex systems and sports : complex systems insights to building effective teams. Cambridge (MA) : NECSI; 2003. PDF téléchargeable à partir du lien : http://necsi .edu/projects/yaneer/SportsBarYam.pdf

35. Bar-Yam Y. Making things work : solving complex problems in a complex world. Cambridge (MA) : NECSI Knowledge Press; 2005.

36. Wheatley M, Frieze D. Using emergence to take social innovation to scale [Internet]. Spokane (WA) : The Berkana Institute; 2006. En ligne à la page : http://margaretwheatley .com/articles/emergence.html

37. Patton MQ. Developmental evaluation : applying complexity concepts to enhance innovation and use ( $1^{\mathrm{e}}$ éd.). New York : The Guilford Press. 2010.

38. Hawe P, Potvin L. What is population health intervention research? Can J Public Health. 2009;100(Suppl 1) : I8-14. 